\title{
Effect of heart rate correction on pre- and post-exercise heart rate variability to predict risk of mortality-an experimental study on the FINCAVAS cohort
}

\section{Paruthi Pradhapan 1,2*, Mika P. Tarvainen ${ }^{3,4}$, Tuomo Nieminen ${ }^{5}$, Rami Lehtinen $^{6}$, Kjell Nikus ${ }^{7,8}$, Terho Lehtimäki ${ }^{7,9}$, Mika Kähönen ${ }^{710}$ and Jari Viik ${ }^{1,2}$}

${ }^{1}$ Department of Electronics and Communication Engineering, Tampere University of Technology, Tampere, Finland

${ }^{2}$ BioMediTech, Tampere, Finland

${ }^{3}$ Department of Applied Physics, University of Eastern Finland, Kuopio, Finland

${ }^{4}$ Department of Clinical Physiology and Nuclear Medicine, Kuopio University Hospital, Kuopio, Finland

${ }^{5}$ Heart and Lung Centre, Helsinki University Central Hospital, Helsinki, Finland

- Tampere Polytechnic, University of Applied Sciences, Tampere, Finland

7 School of Medicine, University of Tampere, Tampere, Finland

${ }^{8}$ Heart Centre, Department of Cardio-Thoracic Surgery, Tampere University Hospital, Tampere, Finland

${ }^{9}$ Fimlab Laboratories, Department of Clinical Chemistry, Tampere, Finland

${ }^{10}$ Department of Clinical Physiology, Tampere University Hospital, Tampere, Finland

Edited by:

Jerzy Sacha, Regional Medical

Center, Poland

\section{Reviewed by:}

Antti M. Kiviniemi, Verve, Finland

Jerzy Sacha, Regional Medical

Center, Poland

*Correspondence:

Paruthi Pradhapan, Department of Electronics and Communication Engineering, Tampere University of Technology, Korkeakoulunkatu 10, Tampere Fl-33720, Finland e-mail: paruthi.pradhapan@tut.fi
The non-linear inverse relationship between RR-intervals and heart rate (HR) contributes significantly to the heart rate variability (HRV) parameters and their performance in mortality prediction. To determine the level of influence HR exerts over HRV parameters' prognostic power, we studied the predictive performance for different HR levels by applying eight correction procedures, multiplying or dividing HRV parameters by the mean RR-interval $\left(R R_{a v g}\right)$ to the power 0.5-16. Data collected from 1288 patients in The Finnish Cardiovascular Study (FINCAVAS), who satisfied the inclusion criteria, was used for the analyses. HRV parameters (RMSSD, VLF Power and LF Power) were calculated from 2-min segment in the rest phase before exercise and 2-min recovery period immediately after peak exercise. Area under the receiver operating characteristic curve (AUC) was used to determine the predictive performance for each parameter with and without HR corrections in rest and recovery phases. The division of HRV parameters by segment's $\mathrm{RR}_{\mathrm{avg}}$ to the power $2\left(H R V_{\text {DIV-2}}\right)$ showed the highest predictive performance under the rest phase (RMSSD: 0.67/0.66; VLF Power: 0.70/0.62; LF Power: 0.79/0.65; cardiac mortality/non-cardiac mortality) with minimum correlation to $\mathrm{HR}$ ( $r=-0.15$ to 0.15$)$. In the recovery phase, Kaplan-Meier (KM) survival analysis revealed good risk stratification capacity at $\mathrm{HRV}_{D / V-2}$ in both groups (cardiac and non-cardiac mortality). Although higher powers of correction ( $H R V_{\text {DIV-4 }}$ and $\left.H R V_{\text {DIV-8}}\right)$ improved predictive performance during recovery, they induced an increased positive correlation to HR. Thus, we inferred that predictive capacity of HRV during rest and recovery is augmented when its dependence on HR is weakened by applying appropriate correction procedures.

Keywords: heart rate correction, heart rate variability, receiver operating characteristics, Kaplan-Meier, FINCAVAS

\section{INTRODUCTION}

Heart rate (HR) recovery and heart rate variability (HRV) have been used by researchers for assessing the role of autonomic regulation in predicting all-cause and cardiovascular mortality (Freeman et al., 2006). The prognostic capabilities of HR response to exercise and after exercise have been well-documented (Lauer et al., 1996; Cole et al., 1999; Lipinski et al., 2004; Jouven et al., 2005; Kiviniemi et al., 2011) and reviewed by Freeman et al. (2006). Increased sympathetic and decreased parasympathetic activities have been associated with an enhanced risk of sudden death or the vulnerability to ventricular arrhythmias (Lahiri et al., 2008). Subdued time- and frequency-domain HRV indices have been linked with increased risk of mortality in the Framingham cohort (Tsuji et al., 1994), survivors of acute myocardial infarction (MI) (Kleiger et al., 1990; Kiviniemi et al., 2007) and cardiovascular morbidity and mortality (Zuanetti et al., 1996). However, studies determining the prognostic capacity of exercise induced short-term HRV have been sparse and inconsistent. Leino et al. (2010) concluded that none of the HRV indices were good predictors of mortality during peak exercise or recovery phase. In a study by Dewey et al. (2007), a greater short-term HRV during recovery post exercise was associated with an increased risk for all-cause and cardiovascular mortality. This is in contrast to observations made in resting HRV, which implies higher RR-interval variability is associated with better prognosis (Dekker et al., 2000; Leino et al., 2010). 
Nieminen et al. (2007) justified that the non-linear inverse relationship between RR interval and HR could be the cause for misinterpretation when comparing subjects with different $\mathrm{HR}$ levels and this has been concurred by other researchers (Chiu et al., 2003; Sacha and Pluta, 2005; Sacha et al., 2005; Virtanen et al., 2007; Bailón et al., 2011). Possible physiological mechanisms involved have also been probed (Perini and Veicteinas, 2003; Goldberger et al., 2006). The non-linear relation between HR and HRV has been addressed by Sacha and Pluta (2008) and correction methods have been suggested to strengthen or weaken the influence of HR (Sacha et al., 2013a; Sacha, 2013). By determining whether decreasing dependence on HR improves the prognostic capacity of HRV, we sought to establish the influence of HR in predicting mortality risk. The aim of our study was to scrutinize these correction techniques and their influence on the predictive capacity of cardiac and non-cardiac mortality in the Finnish Cardiovascular Study (FINCAVAS) cohort.

\section{MATERIALS AND METHODS PATIENT POPULATION AND FOLLOW-UP}

A total of 2212 consecutive patients, who were referred by a physician and willing to undergo exercise stress tests at the Tampere University Hospital, were recruited between 2001 and 2004 for FINCAVAS. Informed consent was obtained from all the participants prior to the interview. Measurements were conducted as stipulated in the Declaration of Helsinki and the study protocol was approved by the Ethical Committee of the Hospital District of Pirkanmaa, Finland. In addition to raw electrocardiograph (ECG), descriptive information, medical history and habitual lifestyle of each patient were recorded. More detailed information regarding the patient population and sample size determination is described elsewhere (Nieminen et al., 2006). Of these, 1288 patients satisfied the inclusion criteria for this study with good quality HRV measurements for at least 2 min during rest phase, immediately prior to exercise, and 2 min during post-exercise recovery immediately after maximum effort.

The follow-up data consisted of information related to causes of death and was collected in 2011. The information for the follow-up was obtained from Causes of Death Register and has been shown to be reliable (Pajunen et al., 2005). The follow-up yielded 66 cardiac deaths and 94 non-cardiac deaths, while the remaining 1128 patients constituted the survival group.

\section{EXERCISE TESTING PROTOCOL}

The prognoses of mortality were analyzed using HRV indices obtained from 2 min segments during rest phase before exercise and 2 min recovery immediately after maximal exercise. Resting ECG was measured in the supine position prior to exercise. The exercise stress test was then performed on a bicycle ergometer with electrical brakes and the Mason-Likar modified lead system

Table 1 | Baseline characteristics of the study population, classified into survival, cardiac, and non-cardiac mortality groups.

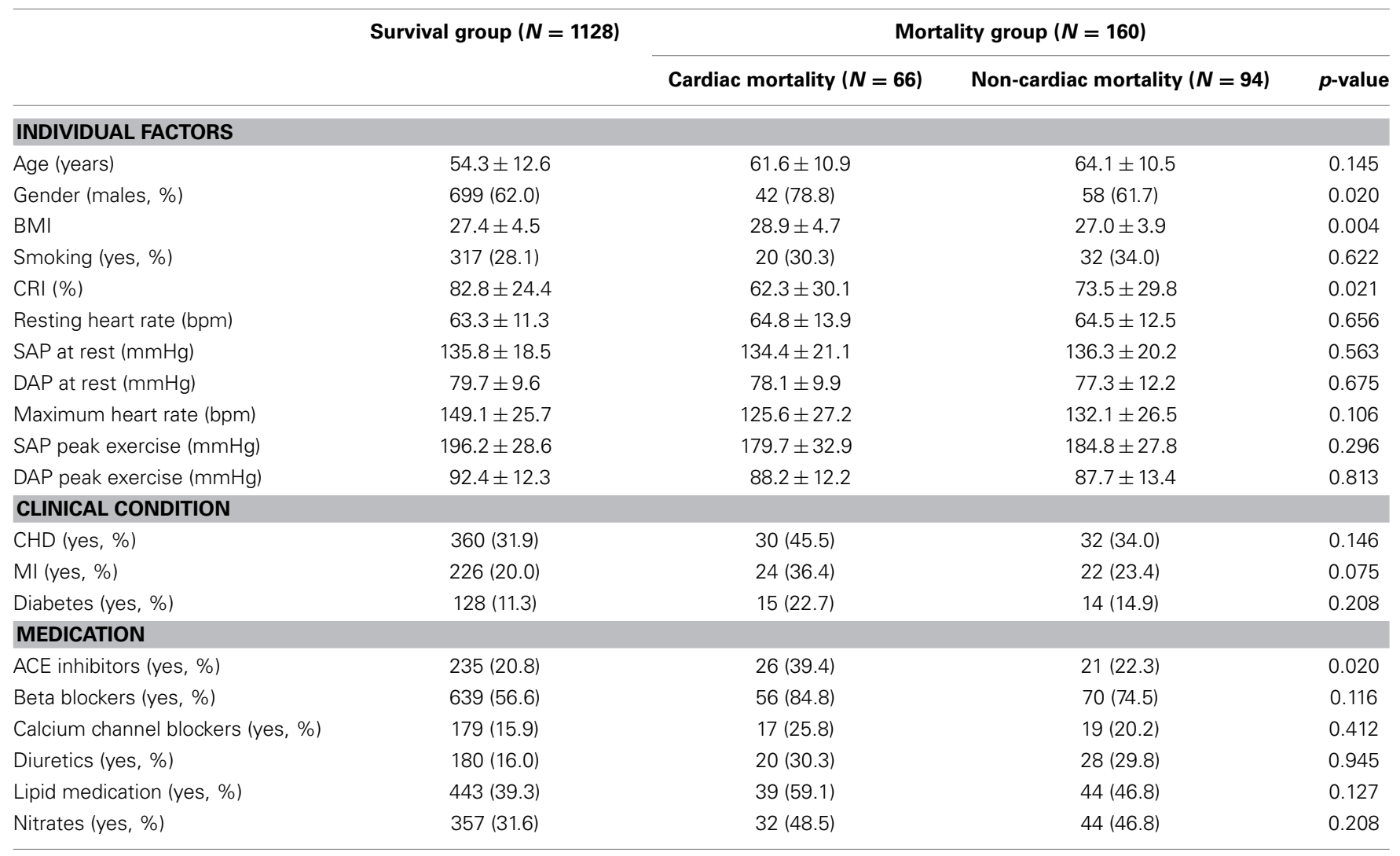

Values are expressed as Mean $\pm S D$ or number of subjects (\%). BMI, body mass index; CRI, chronotropic response index; SAP, systolic arterial pressure; DAP, diastolic arterial pressure; $\mathrm{CHD}$, coronary heart disease; MI, myocardial infarction. 
(Mason and Likar, 1966) was used for the ECG data acquisition. Initial work load and increments were defined based on patient's age, gender, body mass index (BMI) and physical activity. Starting work load varied between 20 and $30 \mathrm{~W}$ and the stepwise increments ranged between 10 and $30 \mathrm{~W}$ every minute. ECG and HR were measured continuously during the test. Tests were sign- and symptom-limited with the recommended criteria for termination whereas in the case of post-MI patients, the upper limit for HR was set at 120-130 beats per minute (bpm). The chronotropic response index (CRI), which represents the chronotropic response to exercise, was evaluated as $\mathrm{CRI}=100 \times$ (peak HR - resting HR)/(220 - age - resting HR) (Kiviniemi et al., 2011). CRI $<80 \%$ was defined as low reserve capacity (Lauer et al., 1996). Measurement during the recovery phase was performed in the sitting position, immediately after exercise.

\section{HRV MEASUREMENT}

ECG was recorded at a sampling frequency of $500 \mathrm{~Hz}$ using CardioSoft exercise ECG system (Version 4.14, GE Healthcare, Freiburg, Germany) and was analyzed using Modified CASE software (GE Healthcare, Freiburg, Germany). After producing the RR-interval tachogram, the data was preprocessed to remove abnormal intervals and artifacts before they were divided into shorter segments based on the stages of rest and recovery. HRV parameters were determined using the Kubios HRV analysis software (Tarvainen et al., 2014). All intervals were resampled using cubic spline interpolation at $4 \mathrm{~Hz}$. Linear and smoothness prior (smoothing parameter, $\lambda=500$ ) detrending were performed prior to calculating time-domain parameters. The fast Fourier transform (FFT) spectrum was computed with a window width of 240 samples, which corresponds to the length of 1 min segment with $4 \mathrm{~Hz}$ resampling rate. A 50\% overlapping window was used for longer segments. Mean $R R$ intervals $\left(R_{a v g}\right)$ were calculated from each segment individually for the HR correction procedure.

Post-exercise recovery is marked by sympathetic withdrawal and parasympathetic reactivation. Sympathetic activation and attenuated parasympathetic recovery are significantly associated with adverse prognosis. The parameters included for examination were chosen based on previous HRV studies on mortality prediction and its outcomes. Of the spectral measures, low frequency $(0.04-0.15 \mathrm{~Hz}, \mathrm{LF})$ power has been found to increase during exercise in normal subjects and reflects both sympathetic and vagal influences (Malliani et al., 1991). In addition, higher log LF power during recovery significantly predicted increased risk of all-cause and cardiovascular mortality (Dewey et al., 2007). Bigger et al. (1993) demonstrated that spectral measures from short segments $(2-15 \mathrm{~min})$ correlated significantly with those computed using 24-h periods. Bernardi et al. (1996) indicated that very low frequency $(0.0033-0.04 \mathrm{~Hz}, \mathrm{VLF})$ power fluctuations were highly dependent on changes in physical activity, rather than preconceived notion of reflecting autonomic tone and thereby, emphasized the importance of activity as a confounding factor. Therefore, VLF power was evaluated due to its independent risk stratification property for all-cause mortality in patients

Table 2 | Association of individual factors, clinical conditions and medication to cardiac and non-cardiac mortality based on univariate Cox regression.

\begin{tabular}{|c|c|c|c|c|}
\hline & \multicolumn{2}{|c|}{ Cardiac mortality $(N=66)$} & \multicolumn{2}{|c|}{ Non-cardiac mortality $(N=94)$} \\
\hline Age $\geq 60$ years & $2.33(1.43-3.80)$ & $<0.001$ & $3.01(1.98-4.58)$ & $<0.001$ \\
\hline Gender (male) & $2.27(1.26-4.09)$ & $<0.05$ & $0.98(0.65-1.48)$ & 0.91 \\
\hline $\mathrm{BMI} \geq 25$ & $1.40(0.76-2.56)$ & 0.001 & $1.08(0.68-1.73)$ & 0.47 \\
\hline $\mathrm{CRI} \leq 39 \%$ & $4.98(2.76-8.99)$ & $<0.001$ & $2.63(1.43-4.82)$ & $<0.001$ \\
\hline $\mathrm{HR}_{\text {rest }} \geq 80 \mathrm{bpm}$ & $0.59(0.32-1.06)$ & 0.08 & $0.70(0.44-1.17)$ & 0.13 \\
\hline $\mathrm{HR}_{\max } \leq 120 \mathrm{bpm}$ & $3.69(2.27-6.00)$ & $<0.001$ & $2.12(1.37-3.27)$ & $<0.001$ \\
\hline \multicolumn{5}{|l|}{ CLINICAL CONDITIONS } \\
\hline CHD (yes) & $1.72(1.06-2.78)$ & $<0.05$ & $1.05(0.68-1.60)$ & 0.84 \\
\hline \multicolumn{5}{|l|}{ MEDICATION } \\
\hline ACE inhibitors (yes) & $2.37(1.45-3.89)$ & $<0.001$ & $1.06(0.65-1.73)$ & 0.81 \\
\hline Beta blockers (yes) & $3.95(2.02-7.75)$ & $<0.001$ & $2.03(1.28-3.23)$ & $<0.05$ \\
\hline Calcium channel blockers (yes) & $1.76(1.01-3.05)$ & $<0.05$ & $1.29(0.78-2.13)$ & 0.33 \\
\hline Diuretics (yes) & $2.09(1.24-3.54)$ & $<0.05$ & $2.09(1.34-3.25)$ & $<0.05$ \\
\hline Lipid medication (yes) & $2.11(1.29-3.45)$ & $<0.05$ & $1.29(0.86-1.93)$ & 0.22 \\
\hline Nitrates (yes) & $1.87(1.16-3.03)$ & $<0.05$ & $1.70(1.13-2.55)$ & $<0.05$ \\
\hline
\end{tabular}

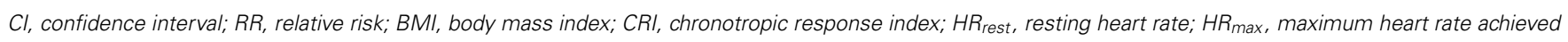
during peak exercise; CHD, coronary heart disease; MI, myocardial infarction. 


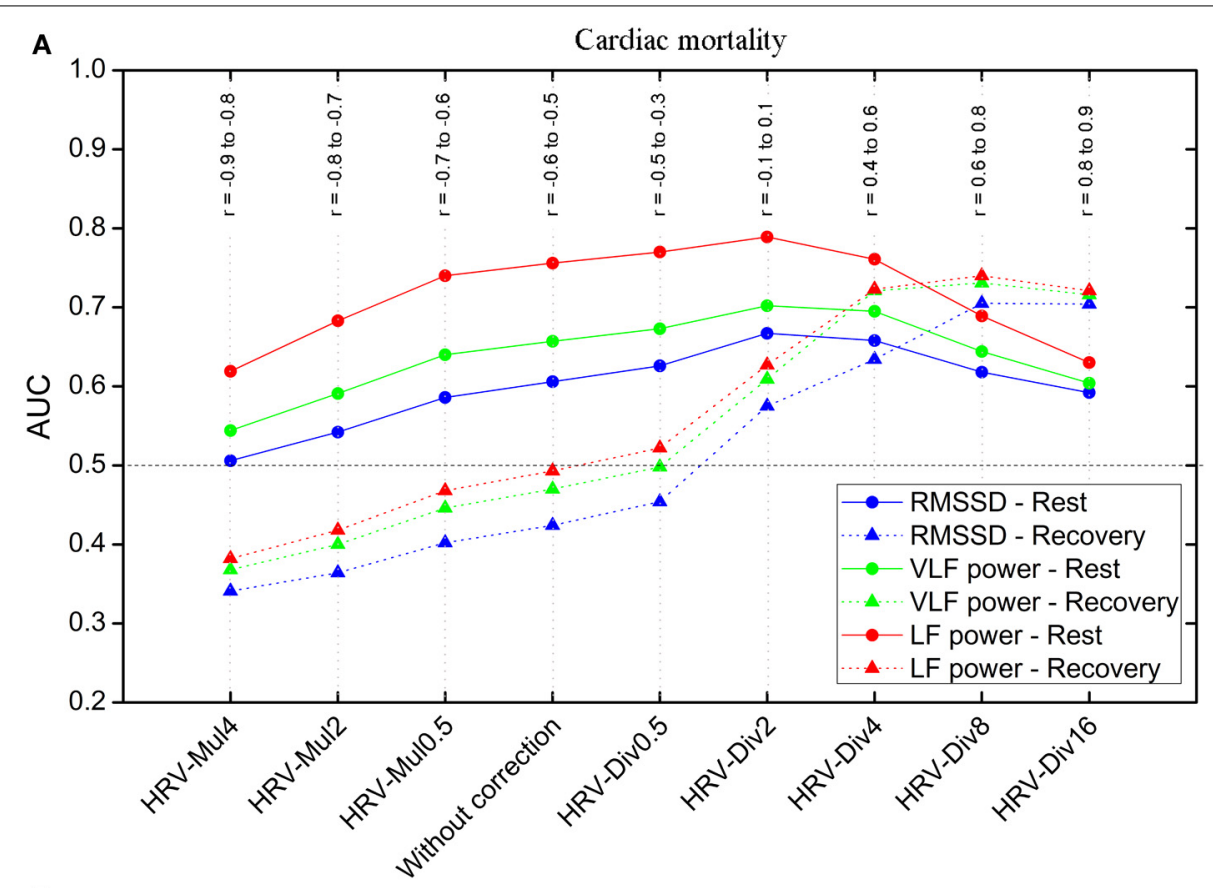

B

Non-cardiac mortality

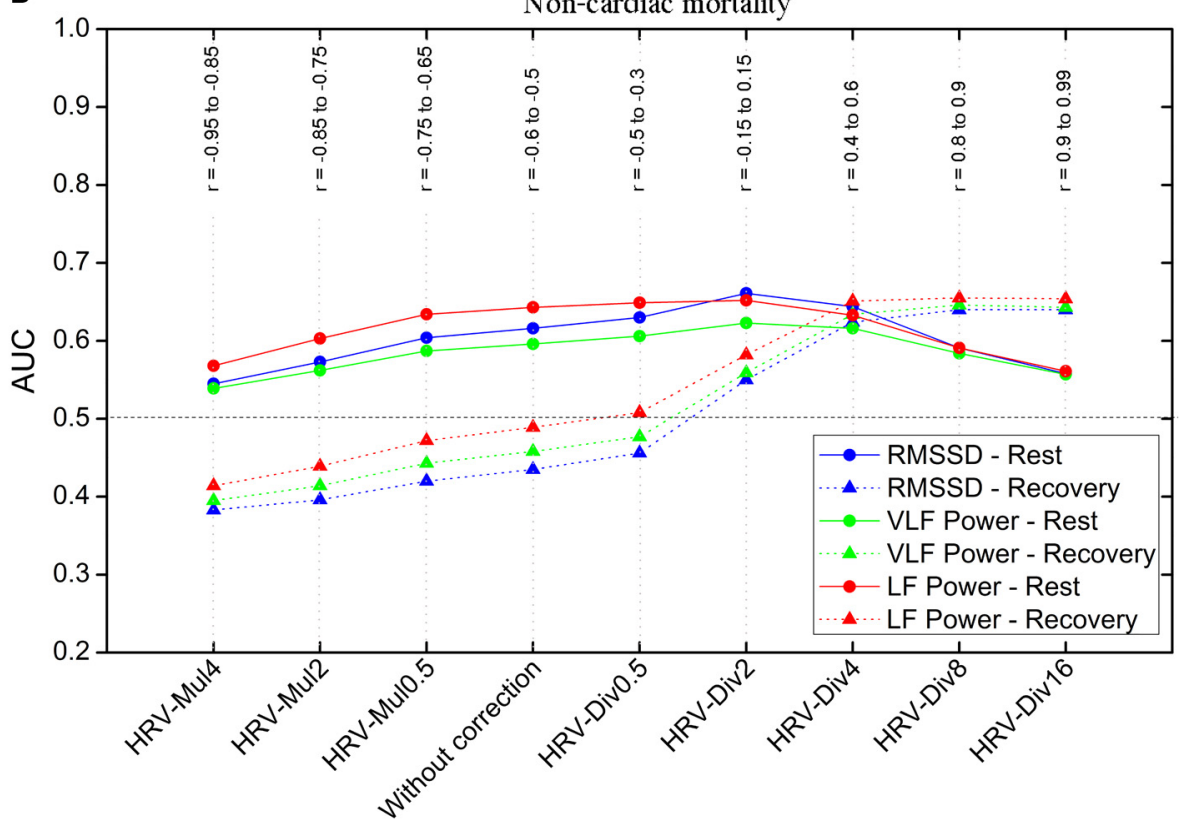

FIGURE 1 | Predictive performance of heart rate variability (HRV) parameters for: (A) cardiac mortality and (B) non-cardiac mortality groups. Area under the receiver operating characteristics curves (AUC) and correlation coefficients (r), between HRV parameters and HR, for different correction methods during rest and recovery after exercise. AUC $>0.5$ indicates that higher heart rate variability (HRV) is associated with better prognosis and AUC $<0.5$ indicates higher HRV is associated with worse prognosis. with acute MI (Bigger et al., 1993). Although high frequency (0.15-0.4 Hz, HF) power has been frequently used to measure parasympathetic tone in resting HRV, interpreting values during recovery after exercise is complicated due to tonic autonomic activity and residual adrenergic activity (Dewey et al., 2007). Goldberger et al. (2006) demonstrated that short-term (as small as $30 \mathrm{~s}$ windows) root mean squared difference of successive RR intervals (RMSSD), which represents high frequency variations in $\mathrm{HR}$, is adequate for measuring parasympathetic reactivation in recovery phase.

\section{HR CORRECTION}

As described by Sacha et al. (2013a), the HRV dependence on $\mathrm{HR}$ is strengthened or weakened by multiplying or dividing the HRV indices by the corresponding segment's $R_{\mathrm{avg}}$, respectively. In addition to normal determination of HRV indices, 
eight other classes for the indices were assessed in this study:

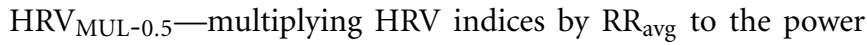
0.5; $\mathrm{HRV}_{\text {MUL-2 }}$-multiplying HRV indices by $\mathrm{RR}_{\mathrm{avg}}$ to the power 2; $\mathrm{HRV}_{\mathrm{MUL}}$-4 - multiplying $\mathrm{HRV}$ indices by $\mathrm{RR}_{\mathrm{avg}}$ to the power 4;

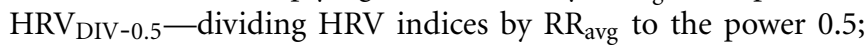

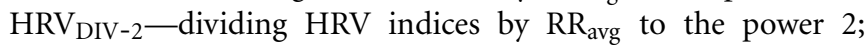
$\mathrm{HRV}_{\text {DIV-4 }}$ - dividing HRV indices by $\mathrm{RR}_{\mathrm{avg}}$ to the power 4; $\mathrm{HRV}_{\text {DIV-8 }}$ - dividing HRV indices by $\mathrm{RR}_{\mathrm{avg}}$ to the power 8; and

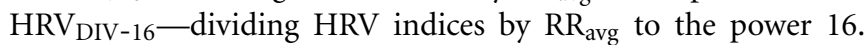
With these classes, different levels of dependence/independence to HR were attained and can be considered significant in determining the contribution of $\mathrm{HR}$ in prognosis of cardiac and non-cardiac mortalities.

\section{STATISTICAL ANALYSES}

The relative risks for cardiac and non-cardiac mortality were assessed for individual characteristics, clinical condition and medication using univariate Cox models. The measure of the predictive power for different HR correction methods for each segment was computed using area under the receiver operating characteristics (ROC) curve. Spearman's rank correlation was performed to determine the degree of correspondence to HR. The cut-off points for Kaplan-Meier (KM) survival analyses were defined from the ROC analyses for each segment. The point of highest overall predictive performance (average of sensitivity and specificity) was chosen as the cut-off to distinguish mortality and survival groups based on HRV observed in the patient population. It has to be noted that these cut-off points were not optimized in order to preserve uniformity during comparisons. The Log-rank chi-square estimates were then used to evaluate the significance of the correction methods based on this classification.

\section{RESULTS}

During the follow-up of the patients who satisfied the inclusion criteria, 66 cardiac deaths were recorded, which included 31 sudden cardiac deaths, with a mean follow-up time of 54 months (min: 4.8 days; max: 99.5 months). 94 patients died of noncardiovascular causes between 1.2 and 110.7 months of follow-up (mean: 60.2 months). The baseline characteristics, clinical conditions and medications used by patients who suffered cardiac and non-cardiac deaths are listed in Table 1.

The univariate Cox regression results for various factors associated with cardiac and non-cardiac mortality are presented in Table 2. The relative risk (RR) of cardiac death was significantly higher in males $[R R=2.27,95 \%$ confidence interval $(C I)=$ $1.26-4.09, p<0.05]$. Age $\geq 60$ years was a risk factor for cardiac $(R R=2.33,95 \% C I=1.43-3.80, p<0.001)$ and non-cardiac $(R R=3.01,95 \% C I=1.98-4.58, p<0.001)$ mortality. Clinical conditions were significantly associated with risk of cardiac death. Medication such as ACE inhibitors $(R R=2.37,95 \% C I=1.45-$ 3.89) and beta blockers $(R R=3.95,95 \% C I=2.02-7.75)$ were significantly associated with increased risk of cardiac mortality $(p<0.001)$.

The area under the ROC curve (AUC) for HR was found to be $0.57 / 0.70$ (rest/recovery) for cardiac mortality and $0.53 / 0.64$ for non-cardiac mortality, implying that HR is a better predictor during recovery than during rest phase. The AUC for RMSSD, VLF and LF power, calculated under different correction methods during rest and recovery phases are presented in Figures 1A,B. Correlation with HR ( $r$, presented in Figure 1) indicated increasing dependence or independence of HRV to HR, based on the method of correction used. AUC $>0.5$ suggested that higher HRV are indicative of better prognosis. $\mathrm{HRV}_{\mathrm{DIV}-2}$, which revealed minimum correlation to $\mathrm{HR}$, was the best predictor for both outcomes (cardiac and non-cardiac mortality) in the rest phase. However, during recovery, higher standard HRV (i.e., HRV without correction) was associated with worse prognosis (AUC < 0.5), as seen in Figure 1. In addition, similar associations were observed for HRV parameters multiplied by different powers of $\mathrm{RR}_{\mathrm{avg}}\left(\mathrm{HRV}_{\mathrm{MUL}-0.5}, \mathrm{HRV}_{\mathrm{MUL}}\right.$, and $\left.\mathrm{HRV}_{\mathrm{MUL}-4}\right)$. Conversely, after division by higher powers of $R_{\mathrm{avg}}$ (i.e., for $H R V_{\mathrm{DIV}-2}$, $\mathrm{HRV}_{\text {DIV-4}}, \mathrm{HRV}_{\text {DIV-8}}$, and HRV $\left.\mathrm{HIV}_{16}\right)$, higher HRV was associated with better prognosis (AUC > 0.5). Though higher orders of correction resulted in better predictive capacity, it also induced moderate/strong positive correlation to $\mathrm{HR}$ (in the case of $\mathrm{HRV}_{\text {DIV-4}}, \mathrm{HRV}_{\text {DIV-8}}$, and HRV $\mathrm{HIV}_{\text {-16 }}$ ).

These results were further corroborated by KM survival analysis. Log-rank estimates at different degrees of correction for both cardiac and non-cardiac mortality are presented in Table 3. $\mathrm{HRV}_{\text {MUL-4 }}$ and HRV $\mathrm{HIV}_{16}$ were excluded due to their very strong correlation to HR. Mortality prediction was most significant for $\mathrm{HRV}_{\text {DIV-2 }}$ in the rest phase. During recovery, the division of HRV by higher powers of $\mathrm{RR}_{\mathrm{avg}}$ resulted in better risk stratification for cardiac and non-cardiac deaths. Although $\mathrm{HRV}_{\mathrm{DIV}-4}$ and $\mathrm{HRV}_{\text {DIV-8 }}$ exhibited better predictive powers during recovery, the HRV indices exhibited strong positive correlation to $\mathrm{HR}(r=0.6$

Table 3 | Chi-square values for Kaplan-Meier analyses under different heart rate correction methods for cardiac and non-cardiac mortality.

\begin{tabular}{llclll}
\hline Parameter & $H_{R V}$ MUL-2 & $\begin{array}{c}\text { Without } \\
\text { correction }\end{array}$ & HRV $_{\text {DIV-2 }}$ & HRV $_{\text {DIV-4 }}$ & HRV $_{\text {DIV-8 }}$ \\
& &
\end{tabular}

\section{CARDIAC MORTALITY}

Two minute resting period prior to exercise

$\begin{array}{lccccc}\text { RMSSD } & 14.10^{* *} & 11.36^{* *} & 43.47^{* *} & 25.22^{* *} & 11.37^{* *} \\ \text { VLF power } & 9.90^{*} & 21.43^{* *} & 27.56^{* *} & 27.84^{* *} & 15.56^{* *} \\ \text { LF power } & 33.84^{* *} & 61.65^{* *} & 75.37^{* *} & 50.60^{* *} & 25.38^{* *} \\ \text { Two minute recovery period post exercise } & & \\ \text { RMSSD } & 15.88^{* *} & 7.93^{* *} & 16.98^{* *} & 30.77^{* *} & 35.84^{* *} \\ \text { VLF power } & 13.56^{* *} & 4.81^{* *} & 21.38^{* *} & 48.48^{* *} & 42.57^{* *} \\ \text { LF power } & 5.50^{*} & 12.72^{* *} & 20.09^{* *} & 41.77^{* *} & 52.71^{* *}\end{array}$

NON-CARDIAC MORTALITY

\section{Two minute resting period prior to exercise}

\begin{tabular}{lccccc} 
RMSSD & $8.97^{*}$ & $16.82^{* *}$ & $26.64^{* *}$ & $21.46^{* *}$ & $10.09^{* *}$ \\
VLF power & $7.63^{*}$ & $15.54^{* *}$ & $19.16^{* *}$ & $19.05^{* *}$ & $10.44^{* *}$ \\
LF power & $16.17^{* *}$ & $21.24^{* *}$ & $24.13^{* *}$ & $17.73^{* *}$ & $12.46^{* *}$ \\
\multicolumn{7}{l}{ Two minute recovery period post exercise } & & \\
RMSSD & $18.60^{* *}$ & $7.83^{*}$ & $4.59^{*}$ & $16.09^{* *}$ & $21.21^{* *}$ \\
VLF power & $9.56^{*}$ & $3.95^{*}$ & $5.08^{*}$ & $19.22^{* *}$ & $29.24^{* *}$ \\
LF power & $4.43^{*}$ & 2.49 & $8.61^{*}$ & $28.39^{* *}$ & $26.01^{* *}$
\end{tabular}

Significance is denoted by ${ }^{*} p<0.05$ and ${ }^{* *} p<0.001$. 


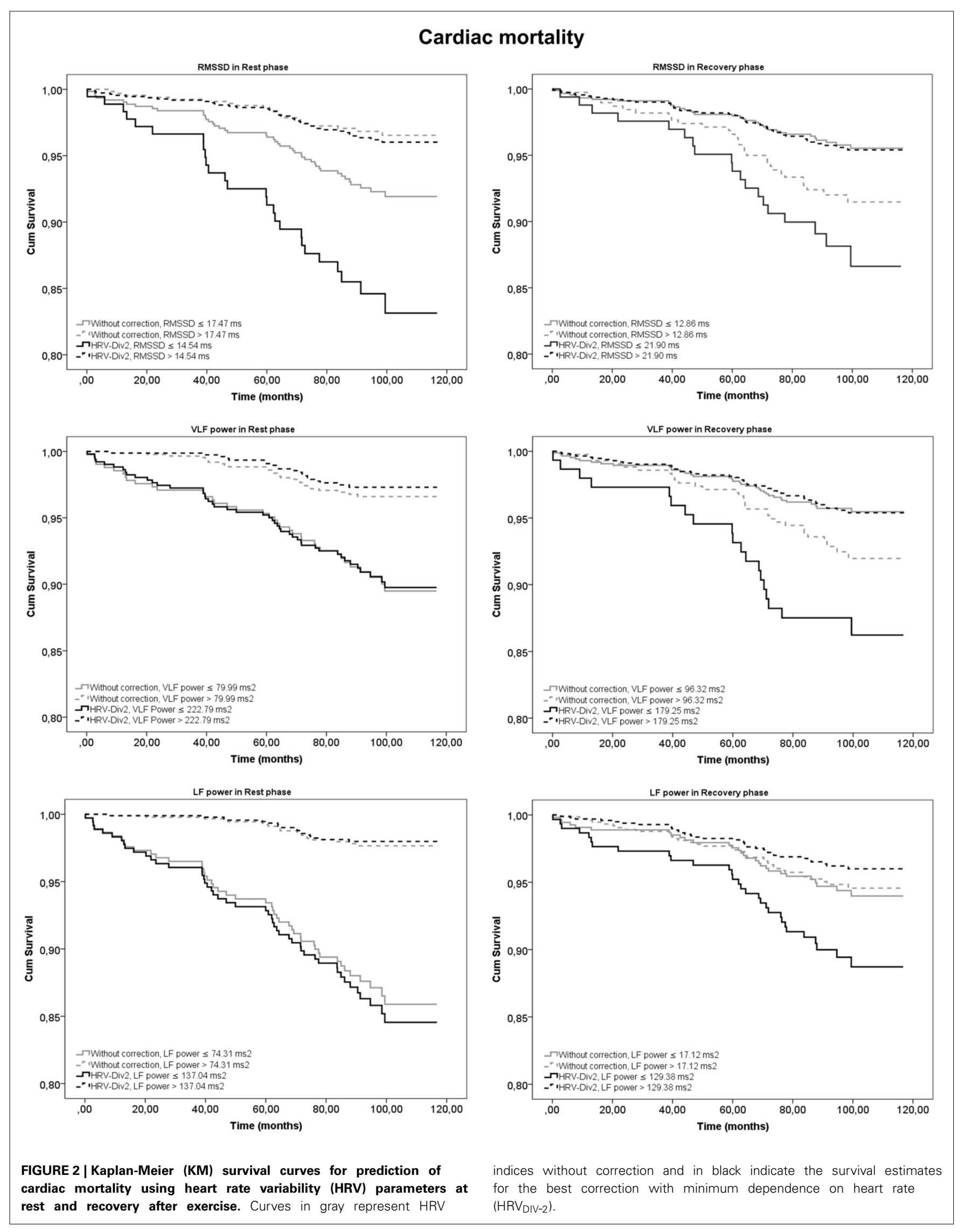




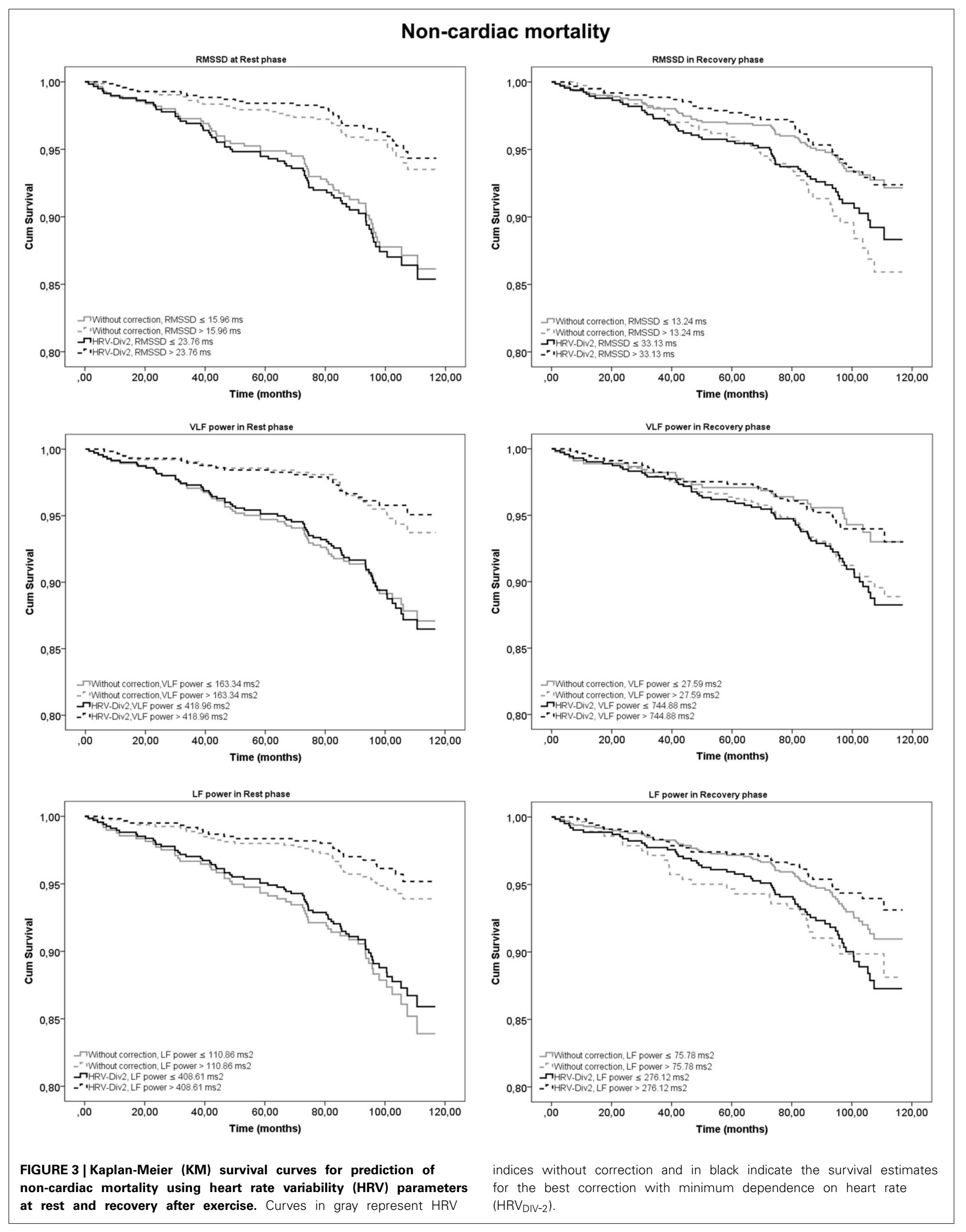


to 0.9 across both groups, as shown in Figure 1) at these correction levels. On the contrary, HRV $\mathrm{DIV}_{2}$ was a good predictor of cardiac $(p<0.001)$ and non-cardiac $(p<0.05)$ mortality during recovery, with minimum influence of $\mathrm{HR}(r=-0.15$ to 0.15$)$. Figures 2, 3 represent the survival curves for $\mathrm{HRV}_{\mathrm{DIV}-2}$ during rest and recovery.

\section{DISCUSSION}

The HRV indices computed from RR-interval measurements correlated with $\mathrm{HR}$ as a result of the non-linear relationship between the RR-interval and instantaneous HR (Chiu et al., 2003; Sacha and Pluta, 2005; Sacha et al., 2005; Nieminen et al., 2007; Virtanen et al., 2007; Bailón et al., 2011). Higher variability during rest and lower variability during recovery were associated with better prognosis, and this corresponds to observations made by Dewey et al. (2007). Our results indicate that the predictive capacity of HRV at rest was highest when the correlation to HR was minimum ( $\mathrm{HRV}_{\mathrm{DIV}-2}, r=-0.15$ to 0.15$)$, suggesting that exclusion of HR influence on resting HRV improved prognostic capacity for cardiac and non-cardiac mortality. Since HR is a poor predictor at rest, removal of HR influence perchance resulted in improved prognostic capacity. On the contrary, HR during recovery phase exhibited significant risk stratification for both outcomes. Thus, increasing HRV's dependence on HR enhanced its predictive capacity (observed in $\mathrm{HRV}_{\mathrm{DIV}-4}$ and $\mathrm{HRV}_{\mathrm{DIV}-8}$ ). However, higher degrees of correction produced moderate/strong positive correlation to HR, similar to observations made by Sacha et al. (2013c), Sacha (2014). To attain true independence, the correction technique that yields HRV least influenced by $\mathrm{HR}$, needs to be identified. In our study, HRV $\mathrm{DIV}_{-2}$ demonstrated improvement in predictability of mortality risk during recovery phase with minimum dependence on HR.

However, conclusive evidence could not be established to distinguish between cardiac and non-cardiac related deaths. This is in contrast to findings by Sacha et al. (2013b), who suggested that increasing the HRV dependence on HR resulted in greater predictive ability for cardiac death and increasing its independence indicated greater predictive power for non-cardiac death. One possible explanation could be that the study population analyzed by Sacha and coworkers comprised only post-MI patients whereas the current study included more heterogeneous patient material.

This study suffered certain limitations. First, the risk factors for individual, clinical conditions and medication were not modeled to determine their contribution toward mortality prediction. By including these variables to the analyses, a more definite conclusion on the cause of mortality could have been established. Second, the patients were not controlled for the type of medication prescribed. The effects of beta blockers and nitrates have been known to affect HR, which could have an effect on the results of HR correction. However, the purpose of the current study was to evaluate the effects of HR correction methods in mortality prediction and therefore, these issues need to be considered in future studies.

\section{CONCLUSION}

The findings of this study indicate that the predictive power of HRV parameters for both cardiac and non-cardiac mortality is augmented when its dependence on HR is weakened during rest and recovery. In addition, when HR is a good predictor, increasing HRV's dependence on HR further enhances the risk stratification for both modes of death.

\section{AUTHOR CONTRIBUTIONS}

The study was conceptualized by Tuomo Nieminen, Kjell Nikus, Terho Lehtimäki, Mika Kähönen, and Jari Viik. Data acquisition and analysis was performed by Paruthi Pradhapan, Mika P. Tarvainen, Rami Lehtinen, and Jari Viik. All authors contributed equally in drafting and revising the manuscript.

\section{ACKNOWLEDGMENTS}

This study was financially supported by Tampere University Hospital Medical fund (Grant 9N035), the Finnish Foundation of Cardiovascular Research and Tampere Tuberculosis Foundation.

\section{REFERENCES}

Bailón, R., Laouini, G., Grao, C., Orini, M., Laguna, P., and Meste, O. (2011). The integral pulse frequency modulation model with time-varying threshold: application to heart rate variability analysis during exercise stress testing. IEEE Trans. Biomed. Eng. 58, 642-652. doi: 10.1109/TBME.2010.2095011

Bernardi, L., Valle, F., Coco, M., Calciati, A., and Sleight, P. (1996). Physical activity influences heart rate variability and very-low frequency components in Holter electrocardiograms. Cardiovasc. Res. 32, 234-237. doi: 10.1016/00086363(96)00081-8

Bigger, J. T. Jr., Fleiss, J. L., Rolnitzky, L. M., and Steinman, R. C. (1993). Frequency domain measures of heart period variability to assess risk late after myocardial infarction. J. Am. Coll. Cardiol. 21, 729-736. doi: 10.1016/0735-1097(93) 90106-B

Chiu, H., Wang, T., Huang, L., Tso, H., and Kao, T. (2003). The influence of mean heart rate on measures of heart rate variability as markers of autonomic function: a model study. Med. Eng. Phys. 25, 475-481. doi: 10.1016/S13504533(03)00019-5

Cole, C. R., Blackstone, E. H., Pashkow, F. J., Snader, C. E., and Lauer, M. S. (1999). Heart rate recovery immediately after exercise as a predictor of mortality. $N$. Engl. J. Med. 341, 1351-1357. doi: 10.1056/NEJM199910283411804

Dekker, J. M., Crow, R. S., Folsom, A. R., Hannan, P. J., Liao, D., Swenne, C. A., et al. (2000). Low heart rate variability in a 2-minute rhythm predicts risk of coronary heart disease and mortality from several causes: the ARIC Study. Circulation 102, 1239-1244. doi: 10.1161/01.CIR.102.11.1239

Dewey, F. E., Freeman, J. V., Engel, G., Oviedo, R., Abrol, N., Ahmed, N., et al. (2007). Novel predictor of prognosis from exercise stress testing: Heart rate variability response to the exercise treadmill test. Am. Heart J. 153, 281-288. doi: 10.1016/j.ahj.2006.11.001

Freeman, J. V., Dewey, F. E., Hadley, D. M., Myers, J., and Froelicher, V. F. (2006). Autonomic nervous system interaction with the cardiovascular system during exercise. Prog. Cardiovasc. Dis. 48, 342-362. doi: 10.1016/j.pcad.2005.11.003

Goldberger, J. J., Le, F. K., Lahiri, M., Kannankeril, P. J., Ng, J., and Kadish, A. H. (2006). Assessment of parasympathetic reactivation after exercise. Am. J. Physiol. Heart Circ. Physiol. 290, H2446-H2452. doi: 10.1152/ajpheart.01118.2005

Jouven, X., Empana, J. P., Schwartz, P. J., Desnos, M., Courbon, D., and Ducimetière, P. (2005). Heart rate profile during exercise as a predictor of sudden death. N. Engl. J. Med. 352, 1951-1958. doi: 10.1056/NEJMoa043012

Kiviniemi, A. M., Tulppo, M. P., Hautala, A. J., Mäkikallio, T. H., Perkiömäki, J. S., Seppänen, T., et al. (2011). Long-term outcome of patients with chronotropic incompetence after an acute myocardial infarction. Ann. Med. 43, 33-39. doi: $10.3109 / 07853890.2010 .521764$

Kiviniemi, A. M., Tulppo, M. P., Wichterle, D., Hautala, A. J., Tiinanen, S., Seppänen, T., et al. (2007). Novel spectral indexes of heart rate variability as predictors of sudden and non-sudden cardiac death after acute myocardial infarction. Ann. Med. 39, 54-62. doi: 10.1080/07853890600990375

Kleiger, R. E., Miller, J. P., Krone, R. J. and Bigger, J. T. Jr., (1990). The independence of cycle length variability and exercise testing on predicting mortality of patients 
surviving acute myocardial infarction. The Multicenter Postinfarction Research Group. Am. J. Cardiol. 65, 408-411. doi: 10.1016/0002-9149(90)90801-7

Lahiri, M. K., Kannankeril, P. J., and Goldberger, J. J. (2008). Assessment of autonomic function in cardiovascular disease: physiological basis and prognostic implications. J. Am. Coll. Cardiol. 51, 1725-1733. doi: 10.1016/j.jacc.2008.01.038

Lauer, M., Okin, P., Larson, M. G., Evans, J. C., and Levy, D. (1996). Impaired heart rate response to graded exercise: prognostic implications of chronotropic incompetence in the Framingham Heart Study. Circulation 93, 1520-1526. doi: 10.1161/01.CIR.93.8.1520

Leino, J., Virtanen, M., Kähönen, M., Nikus, K., Lehtimäki, T., Kööbi, T., et al. (2010). Exercise-test-related heart rate variability and mortality: the Finnish cardiovascular study. Int. J. Cardiol. 144, 154-155. doi: 10.1016/j.ijcard.2008.12.123

Lipinski, M. J., Vectrovec, G. W., and Froelicher, V. F. (2004). Importance of the first two minutes after exercise treadmill testing in predicting mortality and the presence of coronary artery disease in men. Am. J. Cardiol. 93, 445-449. doi: 10.1016/j.amjcard.2003.10.039

Malliani, A., Pagani, M., Lombardi, F., and Cerutti, S. (1991). Cardiovascular neural regulation explored in the frequency domain. Circulation 84, 1482-1492. doi: 10.1161/01.CIR.84.2.482

Mason, R. E., and Likar, I. (1966). A new system of multiple-lead exercise electrocardiography. Am. Heart J. 71, 196-205. doi: 10.1016/0002-8703(66)90182-7

Nieminen, T., Kähönen, M., Nikus, K., and Viik, J. (2007). Heart rate variability is dependent on the level of heart rate. Am. Heart J. 154:e13. doi: 10.1016/j.ahj.2007.04.050

Nieminen, T., Lehtinen, R., Viik, J., Lehtimäki, T., Niemelä, K., Nikus, K., et al. (2006). The Finnish Cardiovascular Study (FINCAVAS): characterizing patients with high risk of cardiovascular morbidity and mortality. BMC Cardiovasc. Disord. 6:9. doi: 10.1186/1471-2261-6-9

Pajunen, P., Koukkunen, H., Ketonen, M., Jerkkola, T., Immonen-Räihä, P., KärjäKoskenkari, P., et al. (2005). The validity of the finnish hospital discharge register and causes of death register data on coronary heart disease. Eur. J. Cardiovasc. Prev. Rehabil. 12, 132-137. doi: 10.1097/01.hjr.0000140718.09768.ab

Perini, R., and Veicteinas, A. (2003). Heart rate variability and autonomic activity at rest and during exercise in various physiological conditions. Eur. J. Appl. Physiol. 90, 317-325. doi: 10.1007/s00421-003-0953-9

Sacha, J. (2013). Why should one normalize heart rate variability with respect to average heart rate. Front. Physiol. 4: 306. doi: 10.3389/fphys.2013.00306

Sacha, J. (2014). Interaction between heart rate and heart rate variability. Ann. Noninvasive Electrocardiol. 171, e42-e45. doi: 10.1111/anec.12148

Sacha, J., Barabach, S., Statkiewicz-Barabach, G., Sacha, K., Müller, A., Piskorski, J., et al. (2013a). How to strengthen or weaken the HRV dependence on heart rate - Description of the method and its perspectives. Int. J. Cardiol. 168, 1660-1663. doi: 10.1016/j.ijcard.2013.03.038

Sacha, J., Barabach, S., Statkiewicz-Barabach, G., Sacha, K., Müller, A., Piskorski, J., et al. (2013b). How to select patients who will not benefit from ICD therapy by using heart rate and its variability? Int. J. Cardiol. 168, 1655-1658. doi: 10.1016/j.ijcard.2013.03.040
Sacha, J., and Pluta, W. (2005). Different methods of heart rate variability analysis reveal different correlations of heart rate variability spectrum with average heart rate. J. Electrocardiol. 38, 47-53. doi: 10.1016/j.jelectrocard.2004. 09.015

Sacha, J., and Pluta, W. (2008). Alterations of an average heart rate change heart rate variability due to mathematical reasons. Int. J. Cardiol. 128, 444-447. doi: 10.1016/j.ijcard.2007.06.047

Sacha, J., Pluta, W., and Witosa, A. (2005). Which heart rate is more variable: a slow or a fast one? -It depends on the method of heart rate variability analysis. Folia. Cardiol. 12(Suppl. D), 1-4. Available online at: http://www.cardiologyjournal. org/en/ishne/pdf/1.pdf

Sacha, J., Sobon, J., Sacha, K., and Barabach, S. (2013c). Heart rate impact on the reproducibility of the heart rate variability analysis. Int. J. Cardiol. 168, 4257-4259. doi: 10.1016/j.ijcard.2013.04.160

Tarvainen, M. P., Niskanen, J.-P., Lipponen, J. A., Ranta-aho, P. O., and Karjalainen, P. A. (2014). Kubios HRV - Heart rate variability analysis software. Comput. Methods Programs Biomed. 113, 210-220. doi: 10.1016/j.cmpb.2013. 07.024

Tsuji, H., Venditti, F. J. Jr., Manders, E. S., Evans, J. C., Larson, M. G., et al. (1994). Reduced heart rate variability and mortality risk in an elderly cohort: the Framingham Heart Study. Circulation 90, 878-883. doi: 10.1161/01.CIR.90.2.878

Virtanen, M., Kähönen, M., Nieminen, T., Karjalainen, P., Tarvainen, M., Lehtimäki, T., et al. (2007). Heart rate variability derived from exercise ECG in the diagnosis of coronary artery disease. Physiol. Meas. 28, 1189-1200. doi: $10.1088 / 0967-3334 / 28 / 10 / 005$

Zuanetti, G., Neilson, J. M., Latini, R., Santoro, E., Maggioni, A. P., and Ewing, D. J., (1996). Prognostic significance of heart rate variability in post-myocardial infarction patients in the fibrinolytic era. The GISSi-2 results. Circulation 94, 432-436. doi: 10.1161/01.CIR.94.3.432

Conflict of Interest Statement: The authors declare that the research was conducted in the absence of any commercial or financial relationships that could be construed as a potential conflict of interest.

Received: 27 February 2014; accepted: 14 May 2014; published online: 03 June 2014. Citation: Pradhapan P, Tarvainen MP, Nieminen T, Lehtinen R, Nikus K, Lehtimäki T, Kähönen $M$ and Viik J (2014) Effect of heart rate correction on pre- and postexercise heart rate variability to predict risk of mortality — an experimental study on the FINCAVAS cohort. Front. Physiol. 5:208. doi: 10.3389/fphys.2014.00208

This article was submitted to Clinical and Translational Physiology, a section of the journal Frontiers in Physiology.

Copyright (๑) 2014 Pradhapan, Tarvainen, Nieminen, Lehtinen, Nikus, Lehtimäki, Kähönen and Viik. This is an open-access article distributed under the terms of the Creative Commons Attribution License (CC BY). The use, distribution or reproduction in other forums is permitted, provided the original author(s) or licensor are credited and that the original publication in this journal is cited, in accordance with accepted academic practice. No use, distribution or reproduction is permitted which does not comply with these terms. 